\title{
Effects of Sitagliptin on Pancreatic Beta-Cells in Type 2 Diabetes With Sulfonylurea Treatment: A Prospective Randomized Study
}

\author{
Ai Sato ${ }^{a}$, e, f, Masahiro Takei ${ }^{\mathrm{a}, ~ e}$, Kunihide Hiramatsu $^{\mathrm{b}}$, Teiji Takeda ${ }^{\mathrm{c}}$, Takahide Miyamoto ${ }^{\mathrm{d}}$, \\ Masanori Yamazaki ${ }^{a}$, Yoshihiko Sato ${ }^{\text {a }}$, Mitsuhisa Komatsu ${ }^{\mathrm{a}}$
}

\begin{abstract}
Background: This prospective randomized, multicenter, open-label, comparative study was performed to analyze the effects of sitagliptin on glycemic control and maintenance of beta-cell function in patients with poorly controlled type 2 diabetes treated with low-dose glimepiride.
\end{abstract}

Methods: Forty-one patients with type 2 diabetes mellitus treated with low-dose glimepiride ( $\leq 2 \mathrm{mg} /$ day $)$ were prospectively enrolled in this study (age: 20 - 75 years; hemoglobin A1c (HbA1c): 7.49.4\%). The patients were randomized into two groups: the glimepiride $(\mathrm{G})$ group, in which glimepiride dose was increased gradually to 6 $\mathrm{mg} /$ day, and the sitagliptin (S) group, in which sitagliptin was added at a dose of $50 \mathrm{mg} /$ day.

Results: HbA1c level was significantly decreased after 24 weeks, but not 12 weeks, in the G group, while a significant decrease was seen after 12 weeks in the $\mathrm{S}$ group. Although there were no significant differences in HbA1c level at 24 weeks between the two groups $(\mathrm{P}=0.057)$. The overall trend of changes in HbA1c level suggested that the glucose-lowering effects were superior in the S group. Furthermore, a significant change in fasting glucose was seen in the $S$ group, but not in the G group. Glycemic control target was achieved in $36.7 \%$ and $16.7 \%$ patients in the $\mathrm{S}$ group and the G group, respectively. The proinsulin/insulin (P/I) ratio was significantly increased in the $\mathrm{G}$ group, whereas it tended to decrease in the $\mathrm{S}$ group. After 24 weeks of treatment, no significant difference was observed in the P/I ratio between the two groups, whereas a significant difference was noted in the $\Delta \mathrm{P} / \mathrm{I}$ (amount of change). Albuminuria tended to increase in the $\mathrm{G}$ group compared with the $\mathrm{S}$ group.

Manuscript submitted September 20, 2018, accepted October 20, 2018

aDepartment of Diabetes, Endocrinology and Metabolism, Division of Internal Medicine, Shinshu University School of Medicine, Matsumoto, Japan

bOkaya City Hospital, Okaya, Japan

'Takeda Naika Clinic, Azumino, Japan

dMiyamoto Naika Clinic, Matsumoto, Japan

eAS and MT equally contributed to this study

${ }^{f}$ Corresponding Author: Ai Sato, 3-1-1 Asahi, Matsumoto 390-8621, Japan.

Email: aisa@shinshu-u.ac.jp

doi: https://doi.org/10.14740/jocmr3632
Conclusion: The results of the present study suggested that sitagliptin effectively lowered hyperglycemia and that it may have a protective effect on pancreatic beta-cells when combined with a low dose of glimepiride. Therefore, sitagliptin may represent a useful combination therapy with low-dose sulfonylurea, not only for achieving glycemic control but also for protection of pancreatic beta-cells.

Keywords: Beta-cell; Sitagliptin; Glimepiride; Type 2 diabetes mellitus

\section{Introduction}

The high prevalence and rapid increase in numbers of patients with type 2 diabetes mellitus represent major public health concerns, both worldwide and in Japan, because this disease can cause various complications and adversely affect quality of life. Thus, primary and secondary preventive measures against diabetes mellitus are becoming increasingly important. Improvement of lifestyle, such as a diet and exercise therapy, is a cardinal option for the treatment of type 2 diabetes mellitus. With regard to pharmacotherapy, metformin is recommended as the first-line drug for type 2 diabetes mellitus globally, and several treatment options for use in combination with metformin have been reported [1]. On the other hand, there are no definitive guidelines regarding first-line pharmacotherapy in Japan, and the treatment regimen is decided at the discretion of the attending physician taking into consideration the clinical condition of the patient and the mechanisms of action of the drugs [2]. Conventionally, sulfonylurea (SU) is often prescribed as the first-line antihyperglycemic drug in Japan as there were no other alternatives until reevaluation of biguanides, and Japanese type 2 diabetes patients usually do not have severe obesity. Although the main concerns regarding SU treatment are the occurrence of hypoglycemia and undesirable weight gain, $\mathrm{SU}$ is an important therapeutic drug for type 2 diabetes mellitus, as it has been shown to be safe for longterm use; the UK Prospective Diabetes Study showed that SU can suppress the progression of diabetes-related complications, and it is associated with low medical costs [3]. However, SU may cause hypoglycemia when administered in high doses or when administered in elderly patients, and the committees of the Japan Diabetes Association recommended use of low-dose SU 


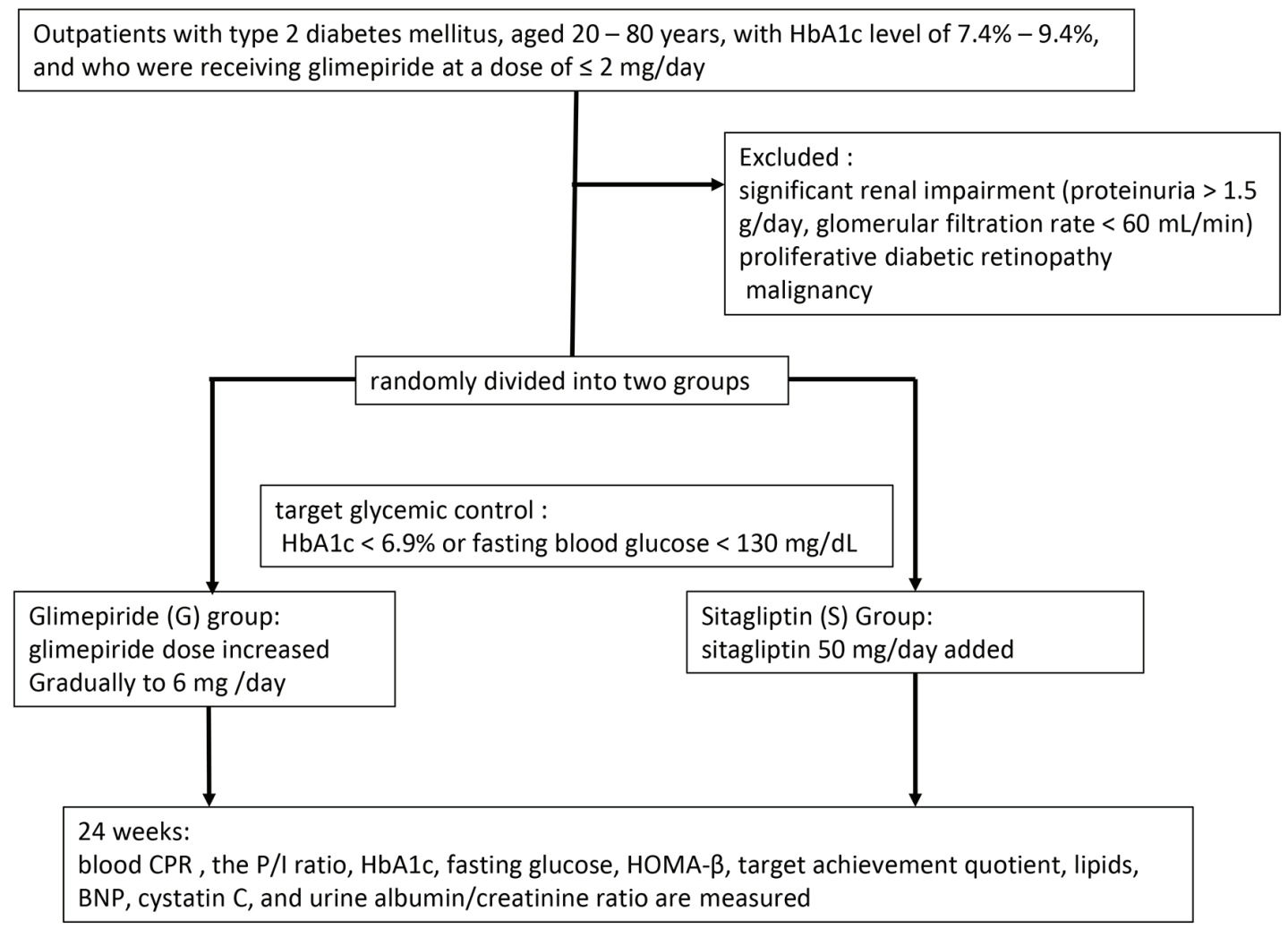

Figure 1. Study protocol.

in such cases [4].

Dipeptidyl peptidase 4 (DPP4) inhibitors, which do not cause hypoglycemia when administered alone, have been increasingly used in Japan based on studies showing sufficient glucose-lowering effects when given in combination with DPP4 inhibitors and various hypoglycemic agents. Sitagliptin, one of the major DPP4 inhibitors, is eliminated primarily by renal excretion and only $16 \%$ of dose excreted as metabolites [5]. On the other hand, glimepiride, a second-generation sulfonylurea, is converted into M1 by CYP2C9 and carboxyl derivatives M2 by cytosolic enzymes [6]. Although several studies have demonstrated the safety of DPP4 inhibitors, studies directly comparing the effects and safety of SU upward titration treatment and combination treatment with low-dose SU and DPP4 inhibitors in Japanese diabetes patients have not been performed. In addition, the protective effect of DPP4 inhibitors on pancreatic beta-cell function has been demonstrated in animal models but not yet in human trials [7]. Therefore, we performed a multicenter, prospective, randomized, open-label, comparative study to examine the differences in glucose-lowering effect and proinsulin/insulin $(\mathrm{P} / \mathrm{I})$ ratio as indicators of beta-cell function, in two different ways to manage hyperglycemia, i.e. SU upward titration therapy and combination therapy consisting of low-dose SU and DPP4 inhibitor.

\section{Materials and Methods}

The study protocol was approved by the ethics committee of
Shinshu University School of Medicine and the study was performed in accordance with the tenets of the Declaration of Helsinki, as amended in Edinburgh in 2000. The study was registered with CMIN-CTR as UMIN000004985. All patients provided informed consent to participate in the study. Outpatients with type 2 diabetes mellitus, aged 20 - 80 years, with hemoglobin A1c (HbA1c) level of 7.4-9.4\%, and receiving glimepiride at a dose of $\leq 2 \mathrm{mg} /$ day, were eligible for inclusion in the study. Patients taking oral antihyperglycemic agents except SU, glinides or DPP4 inhibitors continued taking the drug without changing the dose during the study period. The exclusion criteria were significant renal impairment (proteinuria $>1.5 \mathrm{~g} /$ day, glomerular filtration rate $<60 \mathrm{~mL} / \mathrm{min}$ ), proliferative diabetic retinopathy and malignancy. We enrolled consecutive patients fulfilling the above inclusion criteria and who were regularly treated at the participating institutions for more than 1 year.

The patients were randomly divided into two groups: glimepiride $(\mathrm{G})$ group, in which the glimepiride dose was increased gradually to $6 \mathrm{mg}$; and the sitagliptin (S) group, in which $50 \mathrm{mg} /$ day sitagliptin was added. The target glycemic control was set as $\mathrm{HbA} 1 \mathrm{c}<6.9 \%$ or fasting blood glucose $<$ $130 \mathrm{mg} / \mathrm{dL}$. The increase in glimepiride dose in the G group was determined at the discretion of the chief physician while considering the risk of hypoglycemia. Fasting blood samples were obtained at baseline and after 24 weeks of treatment. The primary outcome measurements were the differences in posttreatment blood C-peptide immunoreactivity (CPR) and P/I 
Table 1. Clinical Characteristics

\begin{tabular}{llll}
\hline & G group $(\mathbf{n}=\mathbf{1 9})$ & S group $(\mathbf{n}=\mathbf{2 2})$ & P value \\
\hline Age (years) & $62.1 \pm 9.0$ & $63.4 \pm 5.9$ & 0.940 \\
Disease duration (years) & $11.9 \pm 4.9$ & $12.9 \pm 6.8$ & 0.699 \\
\hline Body mass index $\left(\mathrm{kg} / \mathrm{m}^{2}\right)$ & $24.6 \pm 3.5$ & $24.0 \pm 2.4$ & 0.432 \\
Systolic blood pressure (mm Hg) & $137 \pm 17$ & $130 \pm 9$ & 0.481 \\
Diastolic blood pressure (mm Hg) & $80 \pm 8$ & $78 \pm 9$ & 0.207 \\
Fasting blood glucose (mg/dL) & $176 \pm 43$ & $175 \pm 34$ & 0.504 \\
Immunoreactive insulin (IU/mL) & $8.9 \pm 5.7$ & $7.2 \pm 4.0$ & 0.687 \\
HbAlc (\%) & $7.8 \pm 0.5$ & $8.0 \pm 0.6$ & 0.423 \\
Urinary albumin (mg/g Cr) & $43.7 \pm 76.2$ & $33.8 \pm 45.2$ & 0.852 \\
Total cholesterol (mg/dL) & $209 \pm 40$ & $202 \pm 35$ & 0.447 \\
HDL-cholesterol (mg/dL) & $56 \pm 14$ & $59 \pm 19$ & 0.339 \\
Triglyceride (mg/dL) & $220 \pm 226$ & $136 \pm 49$ & 0.085 \\
Non-HDL-cholesterol (mg/dL) & $153 \pm 49$ & $143 \pm 34$ & 0.394 \\
\hline
\end{tabular}

G group: glimepiride group; S group: sitagliptin group.

ratio. Secondary outcome measurements included differences in HbA1c, fasting glucose, Homeostatic Model Assessmentbeta-cell function (HOMA- $\beta$ ), target achievement quotient, lipids, B-type natriuretic protein (BNP), cystatin $\mathrm{C}$ and urine albumin/creatinine ratio. The details of the study protocol are shown in Figure 1. All data are presented as means \pm SEM. For statistical analysis, Student's $t$-test, Mann-Whitney $U$ test and $\chi^{2}$ test were performed. In all analyses, $\mathrm{P}<0.05$ was taken to indicate statistical significance.

\section{Results}

The baseline characteristics of the patients are shown in Ta-

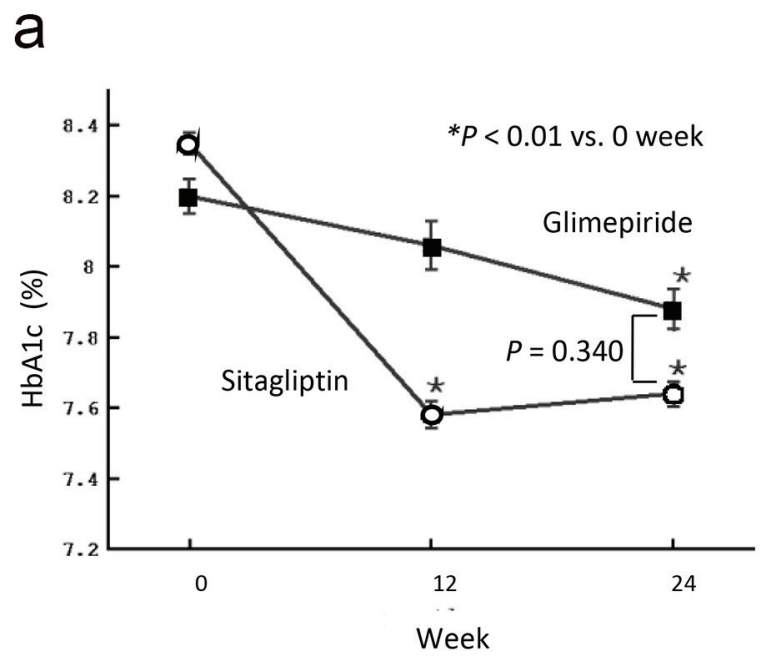

ble 1. Numbers of patients in G group and S group were 19 and 22, respectively. Mean age (62.9 \pm 9.0 years in G group and $63.4 \pm 5.9$ years in $\mathrm{S}$ group, respectively), body mass in$\operatorname{dex}(\mathrm{BMI})\left(24.6 \pm 3.5 \mathrm{~kg} / \mathrm{m}^{2}\right.$ in $\mathrm{G}$ group and $24.0 \pm 2.4 \mathrm{~kg} /$ $\mathrm{m}^{2}$ in $\mathrm{S}$ group, respectively), and other characteristics were no significantly different in baseline clinical or biochemical measurements between the groups. After 24 weeks, HbA1c level decreased significantly from $8.2 \pm 0.5 \%$ to $7.9 \pm 0.6 \%$ in the $\mathrm{G}$ group $(\mathrm{P}<0.01)$ and from $8.4 \pm 0.6 \%$ to $7.6 \pm 0.7 \%$ in the $\mathrm{S}$ group $(\mathrm{P}<0.01)$ (Fig. 2a). However, there was no significant difference in $\mathrm{HbA} 1 \mathrm{c}$ level between the two groups after 24 weeks of treatment. HbA1c level was decreased in the G group at 12 weeks, although the extent of the change was not significant. In the $\mathrm{S}$ group, however, the HbA1c level was

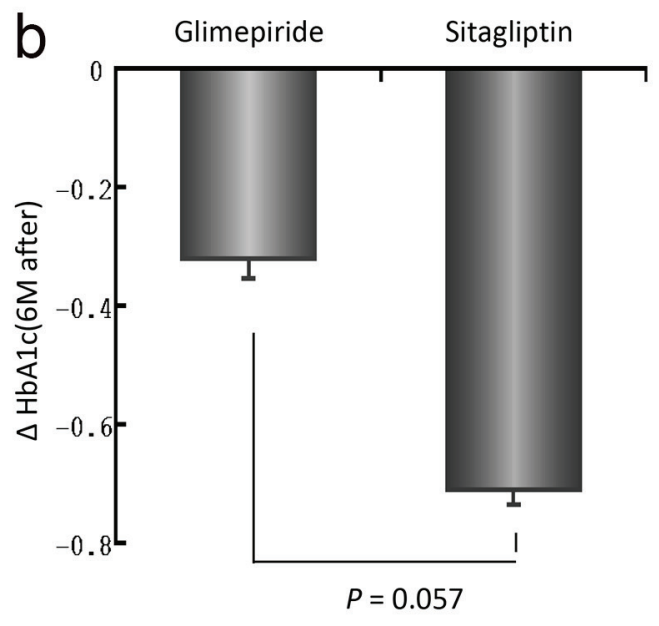

Figure 2. (a) Changes in $\mathrm{HbA} 1 \mathrm{c}$ level over the 24-week treatment period. Actual mean values of $\mathrm{HbA} 1 \mathrm{c}$ in the two groups at 0 , 12 and 24 weeks are shown. The values are expressed as means \pm SEM. A statistical analysis was performed by paired $t$-test. ${ }^{*} \mathrm{P}<0.01$ versus 0 week. (b) The amount of change in $\mathrm{HbA} 1 \mathrm{c}$ from 0 to 24 weeks of the examination. 


\section{a Fasting plasma glucose}

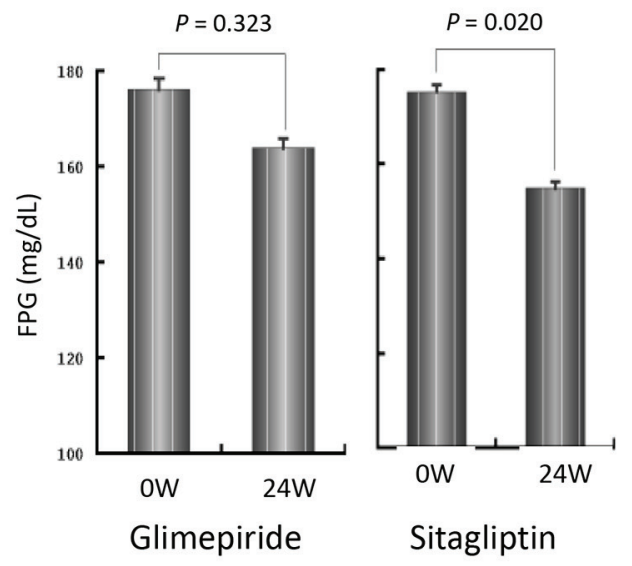

C Proinsulin/Insulin

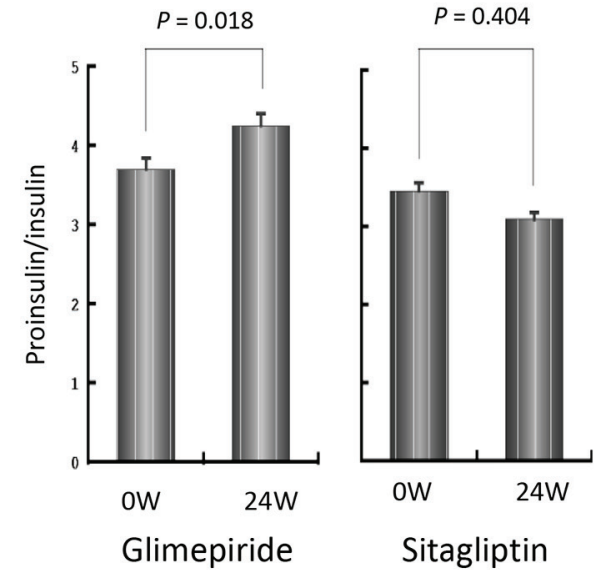

b $\quad$ Fasting IRI

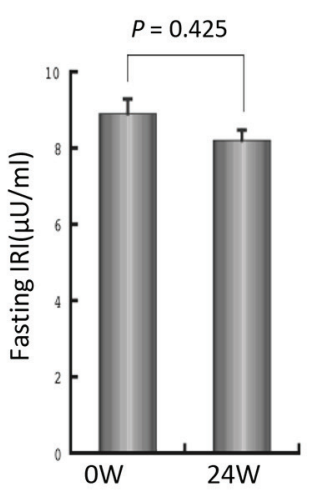

Glimepiride

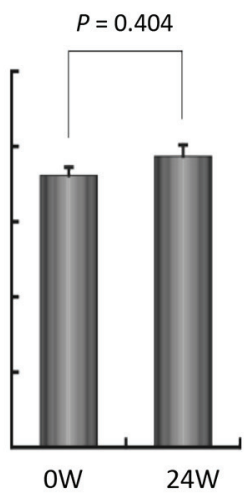

Sitagliptin
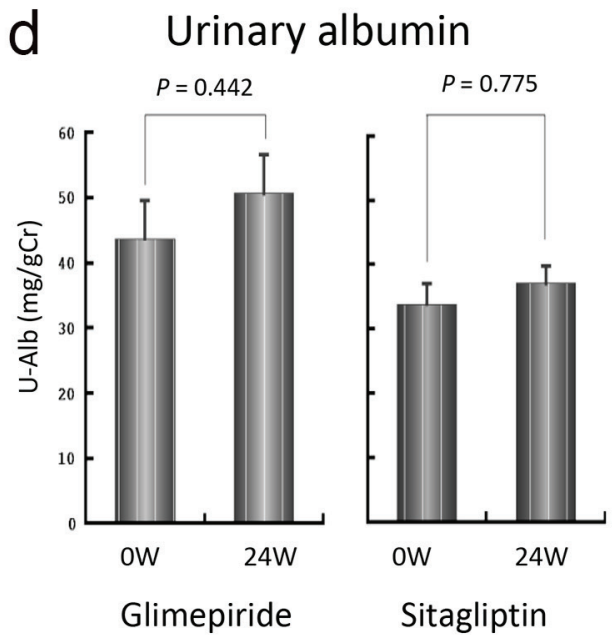

Figure 3. Changes in fasting plasma glucose level (a), fasting insulin concentration (b), proinsulin/insulin ratio (c) and urinary albumin (d) over the 24 -week treatment period. The fasting plasma glucose level was decreased significantly in the $S$ group (a). No significant changes in fasting insulin concentration were observed in either group, although a decreasing tendency was observed in the $G$ group and an increasing tendency was seen in the $S$ group (b). $P / /$ ratio in the $G$ group was significantly increased, while that in the $S$ group was decreased although the difference did not reach statistical significance (c). The urinary albumin excretion tended to increase in the G group, although the difference was not statistically significant (d).

significantly decreased at 12 weeks, and the lowering effect of the treatment remained at 24 weeks. The mean changes in HbA1c $(\triangle \mathrm{HbA} 1 \mathrm{c})$ were $-0.32 \%$ in the G group and $-0.71 \%$ in $\mathrm{S}$ group, but the difference between the two groups was not significant (Fig. 2b).

Figure $3 \mathrm{a}$ shows the changes in fasting plasma glucose level over the 24-week treatment period. While there was no significant change in the $\mathrm{G}$ group compared to baseline, the $\mathrm{S}$ group showed a significant decrease in fasting plasma glucose level from $175 \pm 34 \mathrm{mg} / \mathrm{dL}$ to $155 \pm 33 \mathrm{mg} / \mathrm{dL}$. The target achievement ratio was $16.7 \%$ in the G group and $36.7 \%$ in the $\mathrm{S}$ group.

Figure $3 \mathrm{~b}$ shows the changes in fasting insulin concentration over the 24-week treatment period. While there was no significant change in either group, a decreasing tendency was observed in the G group and an increasing tendency was seen in the $\mathrm{S}$ group.
As shown in Figure $3 \mathrm{c}$, the $\mathrm{P} / \mathrm{I}$ ratio in the $\mathrm{G}$ group was significantly increased from $0.56 \pm 0.33$ at baseline to $0.64 \pm$ 0.34 at 24 weeks $(\mathrm{P}<0.01)$. In contrast, in the $\mathrm{S}$ group, the $\mathrm{P} / \mathrm{I}$ ratio decreased from $0.52 \pm 0.28$ to $0.47 \pm 0.24$, although the difference was not statistically significant. There was no significant difference in $\mathrm{P} / \mathrm{I}$ ratio at 24 weeks between the two groups. However, the change in $\mathrm{P} / \mathrm{I}$ ratio was significantly higher in the $\mathrm{G}$ group than in the $\mathrm{S}$ group ( $\mathrm{G}$ group: +0.08 \pm 0.12 , S group: $-0.05 \pm 0.20, \mathrm{P}<0.01)$. There were no significant differences in CPR, HOMA- $\beta$, lipid profile, BNP or cystatin $\mathrm{C}$ over the 24 -week treatment period or between the two groups (data not shown). As shown in Figure 3d, urinary albumin excretion tended to increase in the G group over time, although this was not statistically significant. Neither group showed any incidences of severe hypoglycemia, defined as hypoglycemic symptoms requiring assistance to recover. One patient in the $\mathrm{G}$ group and two patients in the $\mathrm{S}$ group showed 
mild hypoglycemic symptoms during the study period.

\section{Discussion}

This study demonstrated that adding $50 \mathrm{mg}$ of sitagliptin in patients with poorly controlled type 2 diabetes treated with lowdose glimepiride resulted in comparable glycemic control to treatment with increasing glimepiride dose. The glucose-lowering effects tended to be faster and stronger in the $\mathrm{S}$ group. No severe hypoglycemia was reported during the study. Therefore, this combination appears to be both safe and effective for the treatment of type 2 diabetes.

An interesting result was obtained regarding P/I, which was defined as one of the primary outcomes of this study. The $\mathrm{P} / \mathrm{I}$ ratio was significantly increased after the 24 -week study period in the $\mathrm{G}$ group where the glimepiride dose was increased, while the P/I ratio showed a tendency to decrease in the $\mathrm{S}$ group. Furthermore, the change in $\mathrm{P} / \mathrm{I}$ ratio after the study was significantly higher in the $\mathrm{G}$ group than in the $\mathrm{S}$ group. A high $\mathrm{P} / \mathrm{I}$ ratio suggests that there is a high proportion of proinsulin (pre-stage peptide of insulin) among the secreted insulin, which may indicate beta-cell dysfunction. Recently, Terauchi et al reported that sitagliptin exhibited a lowering effect on P/I, whereas glimepiride had no such effect in Japanese elderly type 2 diabetes patients [8]. Although this head-to-head comparison between $0.5 \mathrm{mg}$ glimepiride and $50 \mathrm{mg}$ sitagliptin for 52 weeks highlighted the usefulness of both drugs in elderly type 2 diabetes patients, our results further suggest benefits associated with combination of low doses of SU and DPP4 inhibitors, which could achieve marked reduction without serious hypoglycemia and may protection beta-cells. In this study, as we increased the dose of SU while taking care not to cause hypoglycemia, the glucose-lowering efficacy was mild and safe. However, with regard to the $\mathrm{P} / \mathrm{I}$ ratio, combination therapy with low-dose $\mathrm{SU}$ and a DPP4 inhibitor may be superior when considering longterm maintenance of pancreatic beta-cells.

Previous studies showed that the glucose-lowering efficacy of DPP4 inhibitors was not inferior to that of SU [8-12]. Moreover, we reported that favorable glycemic control was obtained even if we changed the regimen from high-dose SU to combination therapy with a DPP4 inhibitor and low-dose SU in poorly controlled patients with type 2 diabetes mellitus [13]. Therefore, along with the increased use of DPP4 inhibitors, we do not recommend markedly increasing the dose of SU. However, the long-term safety of DPP4 inhibitors must still be confirmed. In some recent studies, DPP4 inhibitors were shown to be non-inferior to the existing treatment with regard to the development of cardiovascular events over a relatively short term [14-16]. However, it should be noted that the rate of hospitalization for heart failure was increased in the study using the DPP4 inhibitor, saxagliptin [14]. The results with alogliptin, another DPP4 inhibitor, raised doubts regarding cardiac safety in patients with acute coronary syndrome [15]. Nevertheless, it should be emphasized that a similar RCT with sitagliptin contradicted these concerns about increasing rates of heart failure [16]. In addition, DPP4 inhibitor-related pancreatitis is a rare but real event [17].
The present multicenter prospective randomized trial yielded novel findings regarding the changes in $\mathrm{P} / \mathrm{I}$ ratio. DPP4 inhibitors were previously suggested to have favorable effects on $\mathrm{P} / \mathrm{I}$ ratio $[8,18]$. To our knowledge, this is the first report to compare the effects of SU alone versus combination of DPP4 inhibitor and low dose of SU on changes in P/I ratio. No firm conclusions could be drawn regarding the glucose-lowering efficacy and hypoglycemic risk due to the limited number of cases included in this study. In particular, while the target blood glucose level and $\mathrm{HbAlc}$ were set by the upward titration protocol of SU, this treatment regimen resulted in relatively mild hypoglycemia, unlike the findings of a previous overseas study [3], partly because the rate of the dose increase was left to the discretion of each attending physician who considered the risk of hypoglycemia. The observation that $\mathrm{P} / \mathrm{I}$ ratio increased under these conditions in the G group is notable. Although potential limitation of our data is the small number of sample size, it is reasonable to use DPP4 inhibitors in combination with a low dose of SU based on our findings. Taking into account the results of the SAVOR-TIMI study on saxagliptin [14], a difference in urine albumin level between the two groups would have been more likely if greater numbers of cases had been included in this study.

\section{Conclusions}

Combination therapy with DPP4 inhibitor and a low dose of SU showed a rapid and profound effect on glycemic control compared to SU monotherapy. Furthermore, our results suggested that DPP4 inhibitors had a protective effect on beta-cell function.

\section{References}

1. American Diabetes Association. 8. Pharmacologic approaches to glycemic treatment: standards of medical care in diabetes-2018. Diabetes Care. 2018;41(Suppl 1):S73-S85.

2. The Japan Diabetes Association. Diabetes treatment guidebook 2016-2017. 2016; p. 29-32, Bunkodo, Tokyo (in Japanese).

3. Intensive blood-glucose control with sulphonylureas or insulin compared with conventional treatment and risk of complications in patients with type 2 diabetes (UKPDS 33). UK Prospective Diabetes Study (UKPDS) Group. Lancet. 1998;352(9131):837-853.

4. Inagaki N, Iwakura $\mathrm{T}$, Iwamoto $\mathrm{Y}$, Kadowaki $\mathrm{T}$, Seino $\mathrm{S}$, Seino Y. Recommendation from Committee about the proper use of the incretin (GLP-1 receptor agonist and DPP -4 inhibitor). 2011. http://www.jds.or.jp/ (in Japanese).

5. Vincent SH, Reed JR, Bergman AJ, Elmore CS, Zhu B, $\mathrm{Xu} \mathrm{S}$, Ebel D, et al. Metabolism and excretion of the dipeptidyl peptidase 4 inhibitor [14C] sitagliptin in humans. Drug Metab Dispos. 2007;35(4):533-538.

6. Noh K, Kim E, Jeong T, Na M, Baek MC, Liu KH, Park $\mathrm{PH}$, et al. Simultaneous determination of glimepiride and its metabolites in human plasma by liquid chromatogra- 
phy coupled to a tandem mass spectrometry. Arch Pharm Res. 2011;34(12):2073-2078.

7. Seck T, Nauck M, Sheng D, Sunga S, Davies MJ, Stein PP, Kaufman KD, et al. Safety and efficacy of treatment with sitagliptin or glipizide in patients with type 2 diabetes inadequately controlled on metformin: a 2-year study. Int J Clin Pract. 2010;64(5):562-576.

8. Terauchi Y, Yamada Y, Ishida H, Ohsugi M, Kitaoka M, Satoh J, Yabe D, et al. Efficacy and safety of sitagliptin as compared with glimepiride in Japanese patients with type 2 diabetes mellitus aged $\geq 60$ years (START-J trial). Diabetes Obes Metab. 2017;19(8):1188-1192.

9. Goke B, Gallwitz B, Eriksson J, Hellqvist A, Gause-Nilsson I, Investigators DC. Saxagliptin is non-inferior to glipizide in patients with type 2 diabetes mellitus inadequately controlled on metformin alone: a 52-week randomised controlled trial. Int J Clin Pract. 2010;64(12):1619-1631.

10. Matthews DR, Dejager S, Ahren B, Fonseca V, Ferrannini E, Couturier A, Foley JE, et al. Vildagliptin add-on to metformin produces similar efficacy and reduced hypoglycaemic risk compared with glimepiride, with no weight gain: results from a 2-year study. Diabetes Obes Metab. 2010;12(9):780-789.

11. Arechavaleta R, Seck T, Chen Y, Krobot KJ, O'Neill EA, Duran L, Kaufman KD, et al. Efficacy and safety of treatment with sitagliptin or glimepiride in patients with type 2 diabetes inadequately controlled on metformin monotherapy: a randomized, double-blind, non-inferiority trial. Diabetes Obes Metab. 2011;13(2):160-168.
12. Gallwitz B, Rosenstock J, Rauch T, Bhattacharya S, Patel S, von Eynatten M, Dugi KA, et al. 2-year efficacy and safety of linagliptin compared with glimepiride in patients with type 2 diabetes inadequately controlled on metformin: a randomised, double-blind, non-inferiority trial. Lancet. 2012;380(9840):475-483.

13. Ishii H, Ohkubo Y, Takei M, Nishio S, Yamazaki M, Kumagai M, Sato Y, et al. Efficacy of combination therapy with sitagliptin and low-dose glimepiride in Japanese patients with type 2 diabetes. J Clin Med Res. 2014;6(2):127-132.

14. Scirica BM, Bhatt DL, Braunwald E, Steg PG, Davidson J, Hirshberg B, Ohman P, et al. Saxagliptin and cardiovascular outcomes in patients with type 2 diabetes mellitus. N Engl J Med. 2013;369(14):1317-1326.

15. White WB, Cannon CP, Heller SR, Nissen SE, Bergenstal RM, Bakris GL, Perez AT, et al. Alogliptin after acute coronary syndrome in patients with type 2 diabetes. N Engl J Med. 2013;369(14):1327-1335.

16. Green JB, Bethel MA, Armstrong PW, Buse JB, Engel SS, Garg J, Josse R, et al. Effect of sitagliptin on cardiovascular outcomes in type 2 diabetes. N Engl J Med. 2015;373(3):232-242.

17. DeVries JH, Rosenstock J. DPP-4 Inhibitor-Related Pancreatitis: Rare but Real! Diabetes Care. 2017;40(2):161163.

18. Karasik A, Aschner P, Katzeff H, Davies MJ, Stein PP. Sitagliptin, a DPP-4 inhibitor for the treatment of patients with type 2 diabetes: a review of recent clinical trials. Curr Med Res Opin. 2008;24(2):489-496. 